\title{
Continuous and Discrete Transformations of a One-Dimensional Porous Medium Equation
}

\author{
Christodoulos SOPHOCLEOUS \\ Department of Math. and Stat., University of Cyprus, CY-1678 Nicosia, Cyprus \\ E-mail: christod@olympia.mas.ucy.ac.cy
}

Received March 07, 1999; Revised July 09, 1999; Accepted July 12, 1999

\begin{abstract}
We consider the one-dimensional porous medium equation $u_{t}=\left(u^{n} u_{x}\right)_{x}+\frac{\mu}{x} u^{n} u_{x}$. We derive point transformations of a general class that map this equation into itself or into equations of a similar class. In some cases this porous medium equation is connected with well known equations. With the introduction of a new dependent variable this partial differential equation can be equivalently written as a system of two equations. Point transformations are also sought for this auxiliary system. It turns out that in addition to the continuous point transformations that may be derived by Lie's method, a number of discrete transformations are obtained. In some cases the point transformations which are presented here for the single equation and for the auxiliary system form cyclic groups of finite order.
\end{abstract}

\section{Introduction}

Probably the most useful point transformations of partial differential equations (pdes) are those which form a continuous (Lie) group of transformations, each member of which leaves an equation invariant. In addition to possessing continuous groups of symmetries, many pdes also possess discrete symmetries. However, the classical method of deriving the continuous symmetries, which is based on the linear nature of infinitesimal transformations, is not directly applicable to the derivation of groups of discrete symmetries. Such example is given by Kingston and Sophocleous [1] who found that the reciprocal point transformation (double application gives the identity transformation) $x^{\prime}=x / t, t^{\prime}=1 / t$, $u^{\prime}=-u t+x$ leaves the Burger-type equation $u_{t}+u u_{x}+(f(t)-f(1 / t)) u_{x x}=0$ invariant, which is a symmetry additional to the Lie point symmetries obtained from the classical approach.

Point transformations may also be used to link a pde with an equation of a different form. For example, it is sometimes possible to connect an equation to a canonical form for which there is an established theory. Such cases are given in the present paper.

For these reasons it is advantageous to study point transformations directly in finite forms with the ultimate dual aims of finding the complete set of point transformation 
symmetries of pdes and also discovering new links between different equations. A good example, of the approach which is used here, is the work by Sophocleous and Kingston who classify the point transformations of four common classes of one-dimensional non-linear wave equations [2].

We consider the one-dimensional porous medium equation of the form

$$
u_{t}=\left(u^{n} u_{x}\right)_{x}+\frac{\mu}{x} u^{n} u_{x}
$$

We introduce point transformations of a general class and investigate when such transformations map Eq.(1.1) into itself. In addition we classify point transformations that link Eq.(1.1) to different equations but of the same class, where in some cases it turns out to be well known equations. A number of the point transformations obtained form cyclic groups of finite order.

The next section is devoted to the above analysis, where firstly it makes use of a result obtained by Kingston and Sophocleous [3] which is summarised in the following theorem:

Theorem. The point transformation $x^{\prime}=P(x, t, u), t^{\prime}=Q(x, t, u), u^{\prime}=R(x, t, u)$ transforms the pde

$$
u_{t^{\prime}}^{\prime}=H^{\prime}\left(x^{\prime}, t^{\prime}, u^{\prime}, u_{1}^{\prime}, \ldots, u_{n}^{\prime}\right)
$$

to the pde

$$
u_{t}=H\left(x, t, u, u_{1}, \ldots, u_{n}\right)
$$

where $u_{n}=\frac{\partial^{n} u}{\partial x^{n}}, u_{n}^{\prime}=\frac{\partial^{n} u^{\prime}}{\partial x^{\prime n}}, n \geq 2$ and $H$ and $H^{\prime}$ are polynomials (non-negative integral powers) in $u_{1}, \ldots, u_{n}$ and $u_{1}^{\prime}, \ldots, u_{n}^{\prime}$ respectively, if and only if

$$
P=P(x, t), \quad Q=Q(t)
$$

and the functions $H$ and $H^{\prime}$ satisfy the relation

$$
P_{x} R_{u} H=P_{x} Q_{t} H^{\prime}+P_{t} R_{x}+P_{t} R_{u} u_{x}-P_{x} R_{t}
$$

The proof of this theorem can be found in ref. [3].

Equation (1.1) can be written in a conserved form and therefore with an introduction of a new dependent variable may be represented by a system of two pdes. In the Sections 3 and 4 we search for point transformations of a general class that map this system into itself or to a system of the same class. In some cases, such point transformations of this auxiliary system lead to non-local transformations of Eq.(1.1).

Equation (1.1) is a special case of the generalised porous medium equation

$$
u_{t}=\left(u^{n} u_{x}\right)_{x}+f(x) u^{s} u_{x}+g(x) u^{m} .
$$

The classical point symmetries have been presented by Gandarias [4]. Further study, along the lines of this paper, of Eq.(1.3) may therefore be useful. 


\section{Point transformations}

We consider point transformations of the form

$$
x^{\prime}=P(x, t, u), \quad t^{\prime}=Q(x, t, u), \quad u^{\prime}=R(x, t, u)
$$

which transform the pde

$$
u_{t^{\prime}}^{\prime}=\left(f\left(u^{\prime}\right) u_{x^{\prime}}^{\prime}\right)_{x^{\prime}}+g\left(x^{\prime}, u^{\prime}\right) u_{x^{\prime}}^{\prime}
$$

to the pde

$$
u_{t}=\left(F(u) u_{x}\right)_{x}+G(x, u) u_{x}
$$

where $F$ not a constant. We assume that point transformation (2.1) is non-degenerate in the sense that

$$
\frac{\partial(P, Q, R)}{\partial(x, t, u)} \neq 0 \quad \text { and } \quad \frac{\partial(P(x, t, u(x, t), Q(x, t, u(x, t)))}{\partial(x, t)} \neq 0 .
$$

Firstly, we note that each linear transformation of the form

$$
x^{\prime}=c_{1} x+c_{2}, \quad t^{\prime}=c_{3} t+c_{4}, \quad u^{\prime}=c_{5} u+c_{6},
$$

where $c_{i}$ are constants (possibly complex), $c_{1} c_{3} c_{5} \neq 0$, achieves this purpose. That is, Eq. (2.2) is transformed to Eq. (2.3) with the function $F(u)$ defined in terms of $f\left(u^{\prime}\right)$ and $G(x, u)$ in terms of $g\left(x^{\prime}, u^{\prime}\right)$ by

$$
F(u)=c_{3} c_{1}^{-2} f\left(c_{5} u+c_{6}\right), \quad G(x, u)=c_{3} c_{1}^{-1} g\left(c_{1} x+c_{2}, c_{5} u+c_{6}\right),
$$

respectively. Hence, point transformations of the form (2.4) may be filtered out of equations arising in the discussion, without loss of generality, with the understanding that all point transformations obtained may be augmented by Eqs.(2.4) and (2.5).

We now turn into the special case of Eq.(2.3), where $F(u)=u^{n}$ and $G(x, u)=\frac{\mu}{x} u^{n}$,

$$
u_{t}=\left(u^{n} u_{x}\right)_{x}+\frac{\mu}{x} u^{n} u_{x}
$$

The Lie point symmetries of the equation $u_{t}=\left(u^{n}\right)_{x x}+\frac{\mu}{x}\left(u^{n}\right)_{x}$, which is equivalent to Eq.(2.6) with the exception of the case where $n=-1$, have been classified by Gandarias et al [5]. The symmetries of Eq.(2.6) are summarised in the following table, where $(X, T, U)$ are the components of the symmetry generator $X \frac{\partial}{\partial x}+T \frac{\partial}{\partial t}+U \frac{\partial}{\partial u}$ :

\begin{tabular}{lccl}
\hline & $n$ & $\mu$ & $(X, T, U)$ \\
\hline$\Gamma_{1}$ & $\neq 0$ & arbitrary & $(0,1,0)$ \\
$\Gamma_{2}$ & & & $(x, 2 t, 0)$ \\
$\Gamma_{3}$ & & $(0, n t,-u)$ \\
\hline$\Gamma_{4} \neq-2,-1,0$ & $=\frac{3 n+4}{n+2}$ & $\left((n+2) x^{-\frac{n}{n+2}}, 0,-2 x^{-\frac{2(n+1)}{n+2}} u\right)$ \\
\hline$\Gamma_{5}$ & $=-1$ & $=1$ & $(x \ln x, 0,2(\ln x-1) u)$ \\
\hline
\end{tabular}


We search for point transformations of the form (2.1) that map Eq.(2.2) into Eq.(2.6). We note that both of these equations are such that the Theorem, given in the Introduction, can be applied. Therefore we have $Q=Q(t)$ and $P=P(x, t)$. Also we substitute $H=u^{n} u_{x x}+n u^{n-1} u_{x}^{2}+\frac{\mu}{x} u^{n} u_{x}, H^{\prime}=f\left(u^{\prime}\right) u_{x^{\prime} x^{\prime}}^{\prime}+\frac{\mathrm{d} f}{\mathrm{~d} u^{\prime}} u_{x^{\prime}}^{\prime}{ }^{2}+g\left(x^{\prime}, u^{\prime}\right) u_{x^{\prime}}^{\prime}$ into Eq.(1.2) and with the use of the expressions of $u_{x^{\prime}}^{\prime}, u_{x^{\prime} x^{\prime}}^{\prime}[3]$ the relation (1.2) becomes

$$
\begin{aligned}
& x u P_{x} R_{u}\left(u^{n} P_{x}^{2}-Q_{t} f\right) u_{x x} \\
& +x P_{x}\left(n u^{n} P_{x}^{2} R_{u}-u Q_{t} R_{u u} f-u Q_{t} R_{u}^{2} \frac{\mathrm{d} f}{\mathrm{~d} u^{\prime}}\right) u_{x}^{2}+u\left(x Q_{t} P_{x x} R_{u} f-x P_{t} P_{x}^{2} R_{u}\right. \\
& \left.+\mu u^{n} P_{x}^{3} R_{u}-x Q_{t} P_{x}^{2} R_{u} g-2 x Q_{t} P_{x} R_{x u} f-2 x Q_{t} P_{x} R_{x} R_{u} \frac{\mathrm{d} f}{\mathrm{~d} u^{\prime}}\right) u_{x} \\
& +x u\left(Q_{t} P_{x x} R_{x} f-P_{t} P_{x}^{2} R_{x}+P_{x}^{3} R_{t}-Q_{t} P_{x}^{2} R_{x} g-Q_{t} P_{x} R_{x x} f-Q_{t} P_{x} R_{x}^{2} \frac{\mathrm{d} f}{\mathrm{~d} u^{\prime}}\right)=0 .
\end{aligned}
$$

We set, successively, the coefficients of $u_{x x}, u_{x}^{2}, u_{x}$ and the term independent of $u_{x}$ and $u_{x x}$ in Eq.(2.8) equal to zero to obtain four identities. These identities enable the desired point transformations to be derived and ultimately impose restrictions on the functional forms of $Q(t), P(x, t), R(x, t, u), f\left(u^{\prime}\right)$ and $g\left(x^{\prime}, u^{\prime}\right)$. For example, from the first two we deduce that $f=\frac{P_{x}^{2}}{Q_{t}} u^{n}$ and also that $R$ is linear in $u$. From these we conclude that $f\left(u^{\prime}\right)$ is linear in $u^{\prime n}$, but using the transformation (2.4) we can simply write $f\left(u^{\prime}\right)=u^{\prime n}$.

In the following analysis we omit any further calculations. It turns out that this analysis can be split into two cases:(1) $n=-1$; (2) $n \neq-1$. Firstly, we point out that the discrete transformations which correspond to the symmetries $\Gamma_{1}-\Gamma_{3}$ admitted by Eq.(2.6) are of the form (2.4).

Case 1: $(n=-1)$ Equation (2.6) takes the form

$$
u_{t}=\left(\frac{u_{x}}{u}\right)_{x}+\frac{\mu}{x u} u_{x}
$$

If $\mu=1$, Eq.(2.9) is mapped into itself by the transformation

$$
x^{\prime}=x^{k+1}, \quad t^{\prime}=t, \quad u^{\prime}=\frac{1}{(k+1)^{2}} x^{-2 k} u .
$$

This latter transformation is the continuous symmetry which is represented by the symmetry $\Gamma_{5}=x \ln x \frac{\mathrm{d}}{\mathrm{d} x}+2(\ln x-1) u \frac{\mathrm{d}}{\mathrm{d} u}$ admitted by Eq.(2.9).

If we apply transformation (2.10) $N$-times we obtain

$$
x^{(N)}=x^{(k+1)^{N}}, \quad t^{(N)}=t, \quad u^{(N)}=\frac{1}{(k+1)^{2 N}} x^{-2(k+1)^{N}+2} u .
$$

We note that this point transformation forms a cyclic group of order $N$ if $k$ is a root of the equation

$$
(k+1)^{N}-1=0 .
$$

For example, if $N=2$ then $k=-2$ (we omit the obvious solution $k=0$ ) and we get the transformation $x^{\prime}=1 / x, t^{\prime}=t, u^{\prime}=x^{4} u$ which forms a cyclic group of order 2 . In fact, it is trivial to note that $k=0$ and $k=-2$ are the only real roots of equation (2.12). If we 
introduce complex roots of Eq.(2.12), then we may construct cyclic groups of any finite order. For example, the point transformation $x^{\prime}=x^{\mathrm{i}}, t^{\prime}=t, u^{\prime}=-x^{2-2 \mathrm{i}} u$ forms a cyclic group of order 4 .

Furthermore identity (2.8) produces the following three equations that mapped into Eq.(2.9) $(\mu=1)$ by the corresponding discrete transformations:

$$
\begin{aligned}
& u_{t^{\prime}}^{\prime}=\left(\frac{u_{x^{\prime}}^{\prime}}{u^{\prime}}\right)_{x^{\prime}} ; \quad x^{\prime}=k \ln |x|, \quad t^{\prime}=t, \quad u^{\prime}=\frac{1}{k^{2}} u x^{2} ; \\
& u_{t^{\prime}}^{\prime}=\left(\frac{u_{x^{\prime}}^{\prime}}{u^{\prime}}\right)_{x^{\prime}}+\left(\frac{1}{x^{\prime} u^{\prime}}+\frac{1}{2} x^{\prime}\right) u_{x^{\prime}}^{\prime} ; \quad x^{\prime}=\frac{x^{k}}{\sqrt{t}}, \quad t^{\prime}=\ln t, \quad u^{\prime}=\frac{1}{k^{2}} u x^{2(1-k)} ;(2 .) \\
& u_{t^{\prime}}^{\prime}=\left(\frac{u_{x^{\prime}}^{\prime}}{u^{\prime}}\right)_{x^{\prime}}+\frac{1}{2} x^{\prime} u_{x^{\prime}}^{\prime} ; \quad x^{\prime}=\frac{k \ln |x|}{\sqrt{t}}, \quad t^{\prime}=\ln t, \quad u^{\prime}=\frac{1}{k^{2}} u x^{2} .
\end{aligned}
$$

We note from result (2.13) that Eq.(2.9) is connected with a well known nonlinear diffusion equation. Therefore any solution of this diffusion equation can be transformed into a solution of Eq.(2.9) using (2.13). For example, point transformation (2.13) maps the solution $u^{\prime}=2 t^{\prime}\left(x^{\prime 2}+4 t^{\prime 2}\right)^{-1}$ of $u_{t^{\prime}}^{\prime}=\left(u_{x^{\prime}}^{\prime} / u^{\prime}\right)_{x^{\prime}}$ into the solution $u=2 t\left[x^{2}\left(\ln ^{2}|x|+4 t^{2}\right)\right]^{-1}$ of Eq. (2.9).

Finally, if $\mu=0$, the following equation with the corresponding point transformation

$$
u_{t^{\prime}}^{\prime}=\left(\frac{u_{x^{\prime}}^{\prime}}{u^{\prime}}\right)_{x^{\prime}}+\left(\frac{1}{x^{\prime} u^{\prime}}+\frac{1}{2} x^{\prime}\right) u_{x^{\prime}}^{\prime} ; \quad x^{\prime}=\frac{\mathrm{e}^{x}}{\sqrt{t}}, \quad t^{\prime}=\ln t, \quad u^{\prime}=u \mathrm{e}^{-2 x}
$$

is mapped into Eq.(2.9) which has the form of the nonlinear diffusion equation $u_{t}=$ $\left(u_{x} / u\right)_{x}$. Result $(2.16)$ can also be obtained by combining the results (2.13) and (2.14).

Case 2: $(n \neq-1)$ The continuous symmetry which corresponds to the infinitesimal symmetry $\Gamma_{4}=(n+2) x^{-\frac{n}{n+2}} \frac{\partial}{\partial x}-2 u x^{-\frac{2(n+1)}{(n+2)}} \frac{\partial}{\partial u}$ admitted by Eq.(2.6) is the following

$$
x^{\prime}=\left(x^{\frac{2 n+2}{n+2}}+C\right)^{\frac{n+2}{2 n+2}}, \quad t^{\prime}=t, \quad u^{\prime}=u x^{\frac{2}{n+2}}\left(x^{\frac{2 n+2}{n+2}}+C\right)^{-\frac{1}{n+1}}
$$

where also $n \neq-2,0$ and $\mu=\frac{3 n+4}{n+2}$.

In addition to the above result, identity (2.8) produces three more discrete transformations. When $\mu \neq-\frac{n+2}{n}$, the point transformation

$$
x^{\prime}=\frac{2 n+2}{\mu n+n+2} x^{\frac{\mu n+n+2}{2 n+2}}, \quad t^{\prime}=t, \quad u^{\prime}=x^{\frac{\mu-1}{n+1}} u
$$

maps the equation

$$
u_{t^{\prime}}^{\prime}=\left(u^{\prime n} u_{x^{\prime}}^{\prime}\right)_{x^{\prime}}+\lambda \frac{u^{\prime n}}{x^{\prime}} u_{x^{\prime}}^{\prime}
$$

into Eq.(2.6), where $\lambda=\frac{3 n+4-n \mu-2 \mu}{\mu n+n+2}$. If $\lambda=\mu=-\frac{3 n+4}{n}$ then the discrete point transformation $x^{\prime}=\frac{1}{x}, t^{\prime}=t, u^{\prime}=x^{-\frac{4}{n}} u$, which forms a cyclic group of order 2, maps Eq.(2.6) 
into itself. We observe that $\mu=\frac{3 n+4}{n+2}$ implies $\lambda=0$ and Eq.(2.19) becomes the standard nonlinear diffusion equation which is transformed to Eq.(2.6) $(n \neq-2,-1)$ by the transformation

$$
x^{\prime}=\frac{n+2}{2 n+2} x^{\frac{2 n+2}{n+2}}, \quad t^{\prime}=t, \quad u^{\prime}=x^{\frac{2}{n+2}} u .
$$

Also $\mu=0$ implies $\lambda=\frac{3 n+4}{n+2}$ and we obtain the inverse of this latter point transformation which maps Eq.(2.19) into the nonlinear diffusion equation.

When $\mu=-\frac{n+2}{n}$, the point transformation

$$
x^{\prime}=\ln |x|, \quad t^{\prime}=t, \quad u^{\prime}=x^{-\frac{2}{n}} u
$$

maps the equation

$$
u_{t^{\prime}}^{\prime}=\left(u^{\prime n} u_{x^{\prime}}^{\prime}\right)_{x^{\prime}}+2 \frac{n+1}{n} u^{\prime n} u_{x^{\prime}}^{\prime}
$$

into Eq.(2.6). Eq.(2.21) is the Boussinesq equation of hydrology involved in various fields of petroleum technology and ground water hydrology. We observe that when $n=-2$ $(\mu=0)$ Eq.(2.21) is mapped by (2.20) into the well known nonlinear diffusion equation $u_{t}=\left(u^{-2} u_{x}\right)_{x}$. This diffusion equation has a number of properties. For example, admits Lie-Bäcklund transformations and there exists a transformation that maps it into the linear heat equation $u_{t}=u_{x x}[6]$. Also we point out that if $n=-1$ we obtain the result (2.13) of the case 1 .

Finally, when $\mu \neq-\frac{n+2}{n}$, the point transformation

$$
x^{\prime}=\frac{2 n+2}{\mu n+n+2} \frac{x^{\frac{\mu n+n+2}{2 n+2}}}{\sqrt{t}}, \quad t^{\prime}=\ln t, \quad u^{\prime}=x^{\frac{\mu-1}{n+1}} u
$$

maps the equation

$$
u_{t^{\prime}}^{\prime}=\left(u^{\prime n} u_{x^{\prime}}^{\prime}\right)_{x^{\prime}}+\left(\lambda \frac{u^{\prime n}}{x^{\prime}}+\frac{1}{2} x^{\prime}\right) u_{x^{\prime}}^{\prime}
$$

into Eq.(2.6), where $\lambda=\frac{3 n+4-n \mu-2 \mu}{\mu n+n+2}$. In the case where $\mu=-\frac{n+2}{n}$, identity (2.8) gives $n=-1(\mu=1, \lambda=0)$ and we simply reproduce the result $(2.15)$.

\section{Potential transformations}

If we introduce the potential $v$, Eq.(2.6) can be written as a system of two pdes,

$$
v_{x}=u x, \quad v_{t}=x u^{n} u_{x}+\frac{\mu-1}{n+1} u^{n+1},
$$

if $n \neq-1$ and as

$$
v_{x}=u x, \quad v_{t}=\frac{x}{u} u_{x}+(\mu-1) \ln |u|,
$$

if $n=-1$. 
Similarly, if we write

$$
g\left(x^{\prime}, u^{\prime}\right)=\frac{f\left(u^{\prime}\right)+h^{\prime}\left(u^{\prime}\right)}{x^{\prime}},
$$

where $h^{\prime}\left(u^{\prime}\right)=\frac{\mathrm{d} h}{\mathrm{~d} u^{\prime}}$, then Eq.(2.2) can also be written as a system of two pdes

$$
v_{x^{\prime}}^{\prime}=x^{\prime} u^{\prime}, \quad v_{t^{\prime}}^{\prime}=x^{\prime} f\left(u^{\prime}\right) u_{x^{\prime}}^{\prime}+h\left(u^{\prime}\right) .
$$

We also consider the point transformation

$$
x^{\prime}=P(x, t, u, v), \quad t^{\prime}=Q(x, t, u, v), \quad u^{\prime}=R(x, t, u, v), \quad v^{\prime}=S(x, t, u, v)
$$

relating $x, t, u(x, t), v(x, t)$ and $x^{\prime}, t^{\prime}, u^{\prime}\left(x^{\prime}, t^{\prime}\right), v^{\prime}\left(x^{\prime}, t^{\prime}\right)$ and we assume that it is nondegenerate.

It is well known that in many cases pdes which can be written in a conserved form admit nonlocal symmetries, known as potential symmetries [7]. For example, in order to find potential symmetries for Eq.(2.6) we search for point symmetries for the system (3.1) (and (3.2)). If at least one of the infinitesimal generators of the variable $x, t, u$ depends on the potential variable $v$, then the point symmetry of the auxiliary system (3.1) is also a potential symmetry of the Eq.(2.6). Otherwise, the point symmetry of (3.1) projects onto a point symmetry of Eq.(2.6).

The symmetry analysis of the system (3.1) was carried out in the ref. [5]. It was shown that only one symmetry of (3.1) induces a potential symmetry admitted by Eq.(2.6). The rest of the symmetries project onto point symmetries of Eq.(2.6). The potential symmetry occurs if $n=-2$ and $\mu=-\frac{1}{2}$ and is given by

$$
2 x v \frac{\partial}{\partial x}-2\left(x^{2} u^{2}+u v\right) \frac{\partial}{\partial u}+v^{2} \frac{\partial}{\partial v} .
$$

Symmetry (3.6) generates the one-parameter continuous group of transformations

$$
x^{\prime}=\frac{x}{(1-\epsilon v)^{2}}, \quad t^{\prime}=t, \quad u^{\prime}=\frac{u(1-\epsilon v)^{3}}{2 \epsilon x^{2} u+1-\epsilon v}, \quad v^{\prime}=\frac{v}{1-\epsilon v} .
$$

Our goal in this section is to derive point transformations of the class (3.5) which map Eqs.(3.4) into Eqs.(3.1) (and Eqs.(3.4) into Eqs.(3.2)). Using (3.5) and the forms of $u_{x^{\prime}}^{\prime}$, $u_{t^{\prime}}^{\prime}, v_{x^{\prime}}^{\prime}, v_{t^{\prime}}^{\prime}$ in terms of $u_{x}, u_{t}, v_{x}, v_{t}$ and derivatives of $P, Q, R, S$ [8] and eliminating $v_{x}$ and $v_{t}$ from Eqs.(3.1) (or Eqs.(3.2)), Eqs.(3.4) become two identities of the form

$$
E_{1}\left(x, t, u, v, u_{x}, u_{t}\right)=0, \quad E_{2}\left(x, t, u, v, u_{x}, u_{t}\right)=0,
$$

where $x, t, u, v, u_{x}$ and $u_{t}$ are regarded as independent variables and $E_{1}, E_{2}$ are explicit polynomials in $u_{x}$ and $u_{t}$.

Now, the coefficients of $u_{x}^{2}, u_{x}, u_{t}$ in $E_{1}=0$ and $u_{x}^{2}, u_{t}$ in $E_{2}=0$, which must be identically equal to zero, give $P_{u}=Q_{x}=Q_{u}=Q_{v}=S_{u}=0$. Therefore, the point transformation (3.5) can be written in the simplified form

$$
x^{\prime}=P(x, t, v), \quad t^{\prime}=Q(t), \quad u^{\prime}=R(x, t, u, v), \quad v^{\prime}=S(x, t, v) .
$$

To ensure that the point transformations are non-degenerate we require to have

$$
Q_{t} R_{u}\left(P_{x} S_{v}-P_{v} S_{x}\right) \neq 0 .
$$


In addition, we need to have

$$
P_{v}^{2}+R_{v}^{2} \neq 0
$$

because otherwise the point transformations derived are equivalent to point transformations which connect Eq.(2.2) and Eq.(2.6). Transformations that satisfy condition (3.11) shall be called potential transformations.

The restricted forms (3.9) simplify the identities (3.8). For the convenience of the reader we omit any further calculations. We present the three potential transformations obtained: (1) $n=-1$; (2) $n=-2$; (3) $n \neq-2,-1,0$.

Case 1: If $n=-1$ then the point transformation

$$
x^{\prime}=\mathrm{e}^{t+\frac{v}{2}}, \quad t^{\prime}=t, \quad u^{\prime}=\frac{4 \epsilon \mathrm{e}^{-(v+2 t)}}{u x^{2}}, \quad v^{\prime}=2(\epsilon \ln x-t),
$$

transforms the pdes

$$
v_{x^{\prime}}^{\prime}=x^{\prime} u^{\prime}, \quad v_{t^{\prime}}^{\prime}=\epsilon \frac{x^{\prime}}{u^{\prime}} u_{x^{\prime}}^{\prime}+2(\epsilon-1),
$$

where $\epsilon$ is a constant, to the pdes (3.2) where $\mu=1$. Application of the point transformation (3.12) $2 \mathrm{~N}$-times gives

$$
x^{\prime(2 N)}=x^{\epsilon^{N}}, \quad t^{\prime(2 N)}=t, \quad u^{\prime(2 N)}=u x^{2\left(1-\epsilon^{N}\right)}, \quad v^{\prime(2 N)}=\epsilon^{N} v+2\left(\epsilon^{N}-1\right) t .
$$

Therefore transformation (3.12) forms a cyclic group of order $2 N$ if $\epsilon$ is a root of the equation $\epsilon^{N}=1$. Clearly, the only two real roots are $\epsilon=1$ and $\epsilon=-1$ where in these cases transformation (3.12) forms a cyclic group of order 2 and order 4, respectively. If we allow $\epsilon$ to be complex, then we may construct cyclic groups of any even order. For example, if $\epsilon=\mathrm{i}$ then the point transformation $x^{\prime}=\mathrm{e}^{t+v / 2}, t^{\prime}=t, u^{\prime}=\frac{4 \mathrm{i}}{u x^{2} \exp (v+2 t)}$, $v^{\prime}=2(\mathrm{i} \ln x-t)$ forms a cyclic group of order 8 .

If $\epsilon=1$ the reciprocal transformation (3.12) maps Eqs.(3.2) $(\mu=1)$ into itself and therefore we also observe that the point transformation

$$
x^{\prime}=\mathrm{e}^{t+v / 2}, \quad t^{\prime}=t, \quad v^{\prime}=2(\ln x-t)
$$

leaves the integrated form of Eq.(2.9) $\left(u=\frac{v_{x}}{x}\right)$,

$$
v_{t}=\frac{x}{v_{x}} v_{x x}-1
$$

invariant.

Case 2: If $n=-2$ and $\mu=-\frac{1}{2}$ then the point transformation

$$
x^{\prime}=-\frac{x}{v^{2}}, \quad t^{\prime}=t, \quad u^{\prime}=\frac{u v^{3}}{2 x^{2} u-v}, \quad v^{\prime}=\frac{1}{v}
$$

maps the system (3.1) into itself. This latter point transformation forms a cyclic group of order 2. From this result we deduce that the integrated form of Eq.(2.6),

$$
v_{t}=\left(\frac{x}{v_{x}}\right)^{2} v_{x x}+\frac{1}{2} \frac{x}{v_{x}}
$$

remains invariant under the point transformation $x^{\prime}=-x / v^{2}, t^{\prime}=t, v^{\prime}=1 / v$. 
Case 3: If $n \neq-2,-1,0$ and $\mu=\frac{3 n+4}{n+2}$ then the point transformation

$$
x^{\prime}=v^{\frac{n}{2 n+2}}, \quad t^{\prime}=t, \quad u^{\prime}=x^{-\frac{2}{n+2}} v^{\frac{1}{n+1}} u^{-1}, \quad v^{\prime}=\frac{n(n+2)}{4(n+1)^{2}} x^{\frac{2 n+2}{n+2}},
$$

transforms the pdes

$$
v_{x^{\prime}}^{\prime}=x^{\prime} u^{\prime}, \quad v_{t^{\prime}}^{\prime}=\lambda_{1} x^{\prime} u^{\prime-(n+2)} u_{x^{\prime}}^{\prime}+\lambda_{2} u^{\prime-(n+1)}
$$

to the pdes (3.1), where $\lambda_{1}=\frac{n^{2}}{4(n+1)^{2}}$ and $\lambda_{2}=-\frac{n}{2(n+1)^{2}}$. If we use the integrated forms of Eq.(2.6) and Eq.(2.2) we deduce that the point transformation

$$
x^{\prime}=v^{\frac{n}{2 n+2}}, \quad t^{\prime}=t, \quad v^{\prime}=\frac{n(n+2)}{4(n+1)^{2}} x^{\frac{2 n+2}{n+2}}
$$

maps the pde

$$
v_{t^{\prime}}^{\prime}=\lambda_{1}\left(\frac{v_{x^{\prime}}^{\prime}}{x^{\prime}}\right)^{-(n+2)} v_{x^{\prime} x^{\prime}}^{\prime}+\left(\lambda_{2}-\lambda_{1}\right)\left(\frac{v_{x^{\prime}}^{\prime}}{x^{\prime}}\right)^{-(n+1)}
$$

into the pde

$$
v_{t}=\left(\frac{v_{x}}{x}\right)^{n} v_{x x}-\frac{n}{n+2}\left(\frac{v_{x}}{x}\right)^{n+1} .
$$

\section{More potential transformations}

We consider the standard nonlinear diffusion equation

$$
u_{t^{\prime}}^{\prime}=\left(f\left(u^{\prime}\right) u_{x^{\prime}}^{\prime}\right)_{x^{\prime}}
$$

which can be written, with the introduction of the potential variable $v$, as a system of two pdes

$$
v_{x^{\prime}}^{\prime}=u^{\prime}, \quad v_{t^{\prime}}^{\prime}=f\left(u^{\prime}\right) u_{x^{\prime}}^{\prime} .
$$

Here we present point transformations of the form (3.9) which map Eqs.(4.2) into Eqs.(3.1) (or into Eqs.(3.2)).

Case 1: If $n=-1$ the point transformation

$$
x^{\prime}=v+2 t, \quad t^{\prime}=t, \quad u^{\prime}=\frac{c}{u x^{2}}+1, \quad v^{\prime}=v+2 t+c \ln x,
$$

transforms the system (4.2) with $f=\frac{c}{u^{\prime}-1}, c$ a nonzero constant, into the system (3.2) where $\mu=1$.

Case 2: If $n \neq-2,-1$ the point transformation

$$
x^{\prime}=v, \quad t^{\prime}=t, \quad u^{\prime}=x^{-\frac{2}{n+2}} u^{-1}, \quad v^{\prime}=\frac{n+2}{2(n+1)} x^{\frac{2(n+1)}{n+2}},
$$

maps the system (4.2) with $f=u^{\prime-(n+2)}$ into the system (3.1) where $\mu=\frac{3 n+4}{n+2}$. If we use the integrated forms of Eq.(2.6) and Eq.(4.1) we deduce that the point transformation $x^{\prime}=v, t^{\prime}=t, v^{\prime}=\frac{n+2}{2 n+2} x^{\frac{2 n+2}{n+2}}$ maps the pde

$$
v_{t^{\prime}}^{\prime}=v_{x^{\prime}}^{\prime}{ }^{-(n+2)} v_{x^{\prime} x^{\prime}}^{\prime}
$$


into the pde

$$
v_{t}=\left(\frac{v_{x}}{x}\right)^{n} v_{x x}-\frac{n}{n+2}\left(\frac{v_{x}}{x}\right)^{n+1} .
$$

We note that if $n=0$ we obtain the hodograph transformation $x^{\prime}=v, t^{\prime}=t, v^{\prime}=x$ which connects the pde $v_{t^{\prime}}^{\prime}=v_{x^{\prime}}^{\prime-2} v_{x^{\prime} x^{\prime}}^{\prime}$ and the linear heat equation $v_{t}=v_{x x}$. This latter transformation is also a special case of the known general result where this later hodograph transformation connects the equations $v_{t}=F\left(v_{x}\right) v_{x x}$ and $v_{t}=\left(v_{x}\right)^{-2} F\left(1 / v_{x}\right) v_{x x}[9]$.

Furthermore point transformation (4.4) leads to the contact transformation

$$
\mathrm{d} x^{\prime}=u x \mathrm{~d} x+(x u)_{x} \mathrm{~d} t, \quad \mathrm{~d} t^{\prime}=\mathrm{d} t, \quad u^{\prime}=x^{-\frac{2}{n+2}} u^{-1}
$$

which connects the nonlinear diffusion equation $u_{t^{\prime}}^{\prime}=\left(u^{\prime-(n+2)} u_{x^{\prime}}^{\prime}\right)_{x^{\prime}}$ and Eq.(2.6), where $\mu=\frac{3 n+4}{n+2}$. We observe that if $n=0$, transformation (4.5) maps the well known nonlinear diffusion equation $u_{t^{\prime}}^{\prime}=\left(u^{\prime-2} u_{x^{\prime}}^{\prime}\right)_{x^{\prime}}$ into the linear pde $u_{t}=u_{x x}+\frac{2}{x} u_{x}$.

\section{Acknowledgements}

The author would like to thank Prof. M.L. Gandarias for sending him her related work.

\section{References}

[1] Kingston J.G. and Sophocleous C., On Point Transformations of a Generalised Burgers equation, Phys. Lett. A, 1991, V.155, 15-19.

[2] Sophocleous C. and Kingston J.G., Cyclic Symmetries of One-Dimensional Non-Linear Wave Equations, Int. J. Non-Linear Mech., 1999, V.34, 531-543.

[3] Kingston J.G. and Sophocleous C., On Form-Preserving Point Transformations of Partial Differential Equations, J. Phys. A: Math. Gen. 1998, V.31, 1597-1619.

[4] Gandarias M.L., Classical Point Symmetries of a Porous Medium Equation, J. Phys. A: Math. Gen., 1996, V.29, 607-633.

[5] Gandarias M.L., Venero P. and Ramirez J., Similarity Reductions for a Nonlinear Diffusion Equation, J. Nonlin. Math. Phys. 1998, V.5, 234-244.

[6] Bluman G.W. and Kumei S., On the Remarkable Nonlinear Diffusion Equation $(\partial / \partial x)\left[a(u+b)^{-2}\right.$ $(\partial u / \partial x)]-(\partial u / \partial t)=0$, J. Math. Phys., 1980, V.21, 1019-1023.

[7] Bluman G.W. and Kumei S., Symmetries and Differential Equations, Springer, New York, 1989.

[8] Sophocleous C., Linearizing Mappings for Certain Nonlinear Diffusion Equations, J. Phys. A: Math. Gen., 1998 V.31, 6293-6307.

[9] Ibragimov N.H., Lie Group Analysis of Differential Equations, V.1, Chemical Rubber Company, Florida, 1994. 